\title{
Influencia de los instrumentos jurídicos de la Agencia Nacional de Contratación Pública - Colombia Compra Eficiente ${ }^{1}$
}

\author{
Influence of the legal instruments of the Agencia Nacional de \\ Contratación Pública - Colombia Compra Eficiente (National \\ Public Procurement Agency - Colombia Efficient Purchase)
}

\author{
Luis Alfredo Berrío de la Puente iD \\ Especialista en contratación estatal \\ Universidad Externado de Colombia \\ Correo electrónico: luis.berrio85@hotmail.com \\ https://orcid.org/0000-0003-0077-4527
}

\section{Fernando Gutiérrez Ibáñez}

\author{
Abogado Especialista en contratación estatal \\ Asesor jurídico independiente, Valledupar - Colombia \\ Correo electrónico: fernandog213@hotmail.com \\ https://orcid.org/0000-0002-6249-8911
}

\section{Resumen}

El Decreto Ley 4170 de 2011 (Decreto Ley 4170, 2011) estableció, entre otras funciones de la Agencia Nacional de Contratación Pública - Colombia Compra Eficiente, la de construir herramientas orientadas a la organización y articulación de los partícipes en los procesos de

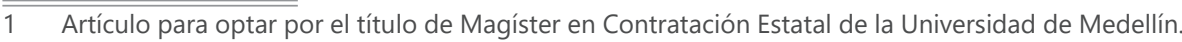


compras y contratación pública, con el fin de lograr una mayor eficiencia, transparencia y optimización de los recursos del Estado.

En el desarrollo de sus funciones, la agencia ha desarrollado instrumentos jurídicos que dilucidan temas específicos y transversales de regulación dentro de la Contratación Pública; sin embargo, estos carecen de las facultades propias y naturales de las normas de derecho común, es por ello que en el presente artículo se analiza el impacto de esos instrumentos en los procesos de contratación pública.

\section{Palabras clave}

Contratación pública, administración, agencia nacional, instrumentos jurídicos.

\section{Abstract}

The Decreto Ley 4170 de 2011 (Law-Decree 4170 of 2011) established, among other functions of the Agencia Nacional de Contratación Pública - Colombia Compra Eficiente, the mandate to build tools aimed at the organization and coordination of the participants of purchase procedures and public procurement, with the aim of achieving higher efficiency, transparency, and optimization of State resources.

While carrying out its functions, the agency has developed legal instruments which elucidate specific and transversal regulatory matters within public procurement; nevertheless, these lack the natural powers of common law rules, which is why this paper analyses the impact of those instruments during public procurement procedures.

\section{Keywords}

Public procurement, administration, national agency, legal instruments..

\section{Introducción}

En el Estado colombiano han sido evidentes carencias y disyuntivas producidas por la falta de una entidad dirigente en materia de contratación pública. Los

\section{Cómo citar este artículo:}

Berrío, L. A., \& Gutiérrez, F. (2020). Influencia de los instrumentos jurídicos de la Agencia Nacional de Contratación Pública - Colombia Compra Eficiente. Revista de la Facultad de Derecho y Ciencias Políticas, 50 (133), pp. 340-355.

doi: http://dx.doi.org/10.18566/rfdcp.v50n133.a05

Recibido: 17 de junio de 2019.

Aprobado: 21 de octubre de 2019. 
continuos cambios de normativas, tales como: decretos, leyes, resoluciones, así como en la doctrina y jurisprudencia han fomentado el desequilibrio e inseguridad en la materia. Esto impide su correcto cumplimiento y ha conllevado una administración defectuosa con respecto a los bienes y recursos del Estado.

Asimismo, la falta de políticas uniformes y de mecanismos de control especializado eficientes son incentivos para que factores como la corrupción y el abuso del derecho se concentren, esto trae consigo la dilapidación y pérdida de los dineros públicos.

Teniendo en cuenta lo anterior, se creó la Agencia Nacional de Contratación -Colombia Compra Eficiente, entidad estatal con la capacidad de brindar asesoría y acompañamiento en los procesos contractuales. Sin embargo, es necesario establecer y destacar el papel y la trascendencia que esta posee, ya que su creación marca un hito para la administración pública y representa un avance en la gestión del Estado.

Para ello se debe analizar la influencia que en la actualidad ejercen los instrumentos jurídicos con que se regulan y establecen lineamientos y políticas de Contratación Pública. Estos son emitidos por la Agencia Nacional de Contratación Pública -Colombia Compra Eficiente, teniendo en cuenta que dichos instrumentos regulan temas propios de la administración pública en materia de adquisición de bienes y servicios, es necesario resaltar que estos no se han derivado del trámite y tránsito legislativo de una norma convencional ni proceden del legislador $\mathrm{y}$, por ende, carecen de fuerza vinculante u obligatoriedad para su aplicación o para exigir el cumplimiento de sus regulaciones. A pesar de lo anterior, han ejercido gran influencia en el desarrollo de procesos de contratación estatal.

\section{Funciones y competencia de la Agencia Nacional de Contratación Pública - Colombia Compra Eficiente}

Con el objetivo de crear políticas sobre compras y contratación pública, el Estado colombiano elaboró lineamientos que sirvieran de apoyo para los gestores de la administración y ejecución de recursos públicos. Para ello se recurrió a la creación de distintas instituciones que se encarguen de promover la medición, inspección y evaluación a fin de suscitar una mayor transparencia en las materias mencionadas. 
Una de las primeras entidades planteada es la Comisión Intersectorial de Contratación Pública, creada por el Decreto 3620 de 2004 (Decreto 3620, 2004), cuyas finalidades principales son: ser el espacio para la elaboración y el debate de decretos reglamentarios que faciliten la conexión, la coordinación y el cumplimiento de las políticas en materia de contratación pública; dirigir la gestión relacionada con la contratación de las entidades públicas enfocada a la reducción de costos en la operación; fortalecer la visibilidad y, por medio de procesos transparente, generar confianza en la gestión contractual pública; precisar medidas para implementar el Sistema Integral de Contratación Electrónica; la cual carece de organización, institucionalidad y de herramientas para satisfacer las necesidades de las que padece el Estado, entre otras.

Sin embargo, tras lo planteado en las Bases del Plan Nacional de Desarrollo 2010-2014 Prosperidad para Todos (Departamento Nacional de Planeación, 2011), se hizo evidente la necesidad de una entidad regente en materia de gestión contractual pública que impulse, relacione, efectúe y realice seguimiento a las políticas que instruyan la actividad contractual del Estado, a la vez que provea herramientas administrativas para permitir la supresión de la corrupción en el uso de los dineros públicos.

Es por ello por lo que a través del Decreto ley 4170 de 2011 (Decreto ley 4170, 2011) se crea la Unidad Administrativa Especial - Agencia Nacional de Contratación Pública - Colombia Compra Eficiente, que se constituye como un organismo técnico especializado de la Rama Ejecutiva del Orden Nacional que se encargará de:

Impulsar políticas y unificar procesos en materia de compras y contratación pública, preparar y suscribir los acuerdos marco de precios de que trata el artículo 2 de la Ley 1150 de 2007 y articular los partícipes de los procesos de compras y contratación pública, con el fin de optimizar los recursos del Estado, hacer coherente la organización y funcionamiento de la Administración Pública para lograr mayor rentabilidad social en el uso de los recursos públicos. (Decreto Ley 4170, 2011)

En su artículo 01 del Decreto 4170 de 2011(Decreto ley 4170, 2011) establece que la Unidad Administrativa será de carácter descentralizada perteneciente a la rama ejecutiva del orden nacional, con personería jurídica y con autonomía administrativa y financiera propias, además se encuentra adscrita al Departamento Nacional de Planeación. 
Por consiguiente, al ser una entidad descentralizada por servicios de la administración, se basa en la necesidad de especialización y tecnificación de las actividades del Estado, sobre todo aquellas de las que el sector central se desprende para permitir un mejor desarrollo de determinadas áreas y funciones.

Al tener personería jurídica propia, tiene también los elementos necesarios que facilitan el cumplimiento de los objetivos propuestos por la agencia, pues cuenta con la facultad de ser sujeto de derechos y obligaciones, lo que permite desarrollar autónomamente su actividad. A su vez, se le concedió un patrimonio propio, con autonomía administrativa y financiera. Estas facultades habilitan la formación de sus dependencias y empleos, además de disponer de reglamentos propios para ejecutar y gestionar su presupuesto.

Para su funcionamiento, el Decreto mencionado estructura a la institución con organismos que se encargan de la dirección y administración de esta. En un primer momento se encuentra el director, quien funge como representante legal y es un funcionario de libre nombramiento y remoción. Se encuentran, además, el Consejo Directivo compuesto por los Ministros de Hacienda, de las TIC, de Comercio y de Transporte; el director del Departamento de Administrativo de la Función Pública y el director de Planeación Nacional, quien presidirá este Consejo Directivo (Decreto Ley 4170, 2011).

Las funciones de este Consejo Directivo están señaladas en el artículo 7 del decreto (Decreto Ley 4170, 2011), concentrándose en la enunciación y aprobación de las políticas propuestas por el Director General de la Agencia, también está a cargo de la vigilar la coordinación entre entidades y de precisar criterios para la instrucción y preparación de los funcionarios. Ambos órganos conforman la Dirección General.

En un segundo momento están órganos que facilitan la gestión de la Agencia, tales como: la Secretaría General, que es responsable de la gestión financiera, contable y de recursos humanos de la entidad. Respecto de las subdirecciones, estas se dividen en: Subdirección de Negocios, encargada de adelantar estudios de mercado, agregar la demanda de las entidades estatales y ofrecer información de calidad sobre el Sistema de Compra Pública a los partícipes de la compra pública. Por su parte, la Subdirección de Información y Desarrollo Tecnológico está encargada de estructurar, implementar, mantener y operar las plataformas tecnológicas para la compra pública y de ofrecer herramientas para que los partícipes de la compra pública tengan información oportuna y 
de calidad para buscar el mayor valor por dinero en la compra pública. Por último, la Subdirección Gestión Contractual es responsable de promover instrumentos jurídicos para orientar a los partícipes, facilitar el proceso de compra y contratación, apoyar en la estructuración legal para consolidar el sistema, al buscar el mayor valor por dinero en la compra pública y atender las consultas de los partícipes sobre el sistema (Colombia Compra Eficiente, s.f.).

Gráfico 1: Organigrama del Consejo Directivo.

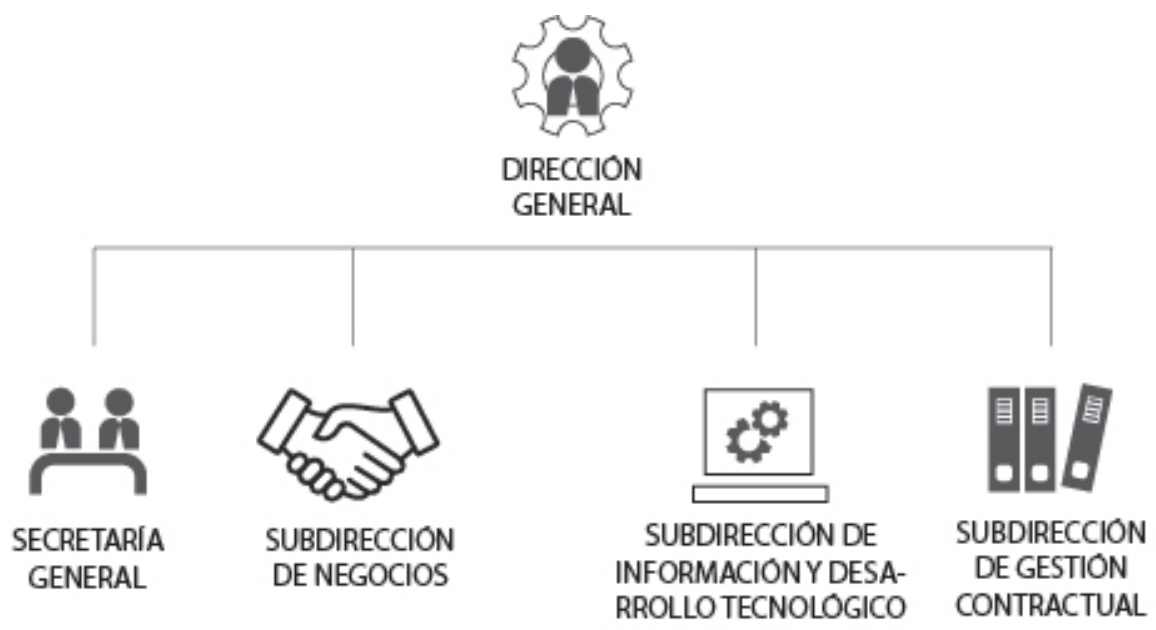

Fuente: https://www.colombiacompra.gov.co/colombia-compra/colombia compra -eficiente/organigrama

Esta entidad tiene como objetivo principal: “desarrollar e impulsar políticas públicas y herramientas, orientadas a la organización y articulación de los partícipes en los procesos de compras y contratación pública con el fin de lograr una mayor eficiencia, transparencia y optimización de los recursos del Estado” (Decreto Ley 4170, 2011).

Para lograr el cumplimiento del objetivo mencionado, en el artículo 3 del decreto ley 4170 (Decreto ley 4170, 2011) se le otorgan varias funciones, principalmente proponer e implementar planes, políticas y normas en materia de contratación pública, así como criterios de racionalización normativa con el fin de lograr una mayor eficiencia, transparencia y optimización de los recursos del Estado. Esto proyecta la implementación de un derecho contractual más ordenado, eficaz, comprensible y estable. 
Simultáneamente, se le encomienda la realización de estudios, diagnósticos y estadísticas en materia de compras y contratación con recursos del Estado, en busca de la efectividad entre la oferta y la demanda en el mercado de compras y contratación pública, es decir, debe propender por una mejor inclusión en el mercado de bienes y servicios. Esto requiere de información del contexto y de las normativas existentes, de tal forma que fundamente las decisiones y conceptos de la agencia con respecto al flujo de los recursos públicos.

También se le otorgó la función de resolver consultas sobre la aplicación de normas de carácter general y expedir circulares externas en materia de compras y contratación pública. Esta es una de las funciones principales de la agencia, ya que era una necesidad urgente dentro de la actividad contractual de las entidades públicas, puesto que ahora los funcionarios públicos dispondrán de una fuente unificada, confiable y accesible en materia de interpretación jurídica contractual pública. Esto estimula la actividad contractual del Estado, al favorecer la transparencia y eficiencia en las entidades públicas (Acosta Ramos, 2013).

Al mismo tiempo, la agencia debe brindar apoyo al Gobierno Nacional en la negociación internacional en materia de compras y contratación pública, especialmente en lo concerniente a los tratados de libre comercio suscritos con los distintos Estados. Esto con el fin de salvaguardar los intereses de los países involucrados y que las negociaciones entre estos sean manejadas de tal forma que el Estado colombiano acceda a determinados bienes y servicios de manera eficiente, para abrir a su vez la oportunidad de que contratistas colombianos puedan proponer sus productos y servicios en el mercado público internacional, en tanto forma de manifestación de las cláusulas de reciprocidad de los tratados de libre comercio suscritos por el Gobierno nacional.

Esta también tiene asignado el diseño, la organización y celebración de los acuerdos marco de precios y demás mecanismos de agregación de demanda, conforme al parágrafo 5 del artículo $2^{\circ}$ de la Ley 1150 de 2007 (Ley 1150, 2007, art. 2, parágrafo 5), debido a que estos presentaron complicaciones para su ejecución, lo que conllevó a la supresión del $\mathrm{SICE}^{2}$, mediante la ley antitrámites. Esta permite al Gobierno ser más eficiente en la elección de proveedores al momento de realizar una operación (Acosta Ramos, 2013).

2 Sistema de Información para la Vigilancia de la Contratación Estatal-SICE, creado por la Ley 598 de 2000 (Ley 598, 2000). 
Además, la agencia tiene la función de desarrollar y administrar el Sistema Electrónico para la Contratación Pública-SECOP y tramitar nuevos desarrollos tecnológicos en los asuntos de su competencia según los lineamientos establecidos por el Consejo Directivo de la entidad.

Entre las distintas funciones se encuentran también: ofrecer apoyo a la administración pública, quien representa la mayor demanda en el mercado contractual; además de desarrollar mecanismos de apoyo a los oferentes, es decir, a empresas contratistas. Esto favorece una mejor y más amplia intervención en los procesos de compras y contratación pública de las entidades estatales (Acosta Ramos, 2013).

A esto se suma que a la agencia creada se le encomendó el deber de divulgar las normas, procedimientos y medios tecnológicos para la actividad contractual estatal, al mismo tiempo de promovery coordinar las capacitaciones necesarias con otras instituciones públicas, a fin de dirigir a los partícipes en el proceso contractual.

Cabe resaltar que las funciones impuestas a la agencia buscan suplir las carencias que poseía la administración del Estado, puesto que no había un organismo rector que se encargara de esta actividad, lo que condujo a una normatividad contractual inconsistente y desordenada. Esta situación generaba inestabilidad e inseguridad, debido a que los distintos ejecutores de estas normas no tenían una normatividad unificada que permitiera una correcta aplicación.

Es menester mencionar que esta es una entidad de carácter nacional, por lo tanto brinda apoyo a las entidades territoriales de los distintos niveles; paralelamente, las políticas, instrumentos, mecanismos y conceptos que esta emite en materia de compras y contratación pública son para cumplimiento de todas las instituciones.

\section{Efectos jurídicos de las directrices emitidas por la Agencia Nacional de Contratación Pública - Colombia Compra Eficiente}

En este marco normativo, la Agencia Nacional de Contratación Pública Colombia Compra Eficiente ha demostrado cumplir con la obligación legal 
de desarrollo e impulso de algunas herramientas en materia de compras y contratación pública, con lo que esta entidad crea instrumentos para una mejor comprensión y aplicación de las normas existentes. Esto asemeja en mayor medida su función a una de carácter consultivo y no a una de carácter imperativo (De Vivero Arciniegas, 2013).

Al reconocer que esta entidad tiene un carácter consultivo se plantea entonces desde la clasificación normativa de Soft Law administrativo ${ }^{3}$ $\mathrm{y}$, por ende, involucra un conjunto de instrumentos jurídicos para que la Administración alcance y materialice eficientemente los fines estatales.

Ahora bien, estas herramientas jurídicas promulgadas por Colombia Compra Eficiente, al ser soft law, tienen las mismas características que estas: la primera es su ausencia de obligatoriedad, esto debido a que conforme a la estructura organizacional y jerárquica de las normas según el modelo kelseniano, los instrumentos se encuentran en la base de la estructura, por lo cual no tienen fuerza coercitiva de acción ni tampoco están al nivel de las normas en sentido estricto o de resoluciones. Por todo esto, su aplicabilidad se convierte en un tema netamente discrecional.

La segunda característica es que estos instrumentos no son esenciales en el ordenamiento jurídico, es decir, la ausencia de estos no afectaría en mayor medida el sistema, ya que el Estado colombiano tiene regulada la actividad de contratación estatal. Y la última característica sería que estas herramientas, en tanto Soft Law, son de carácter auxiliar o interpretativo, debido a que su falta de obligatoriedad, además de su fácil desarrollo y creación, hacen que se desplieguen de una manera más práctica y eficiente aspectos dejados de lado por las normas. Lo que les da una especial característica para regular aspectos diferentes y más profundos que no desarrollan las normas comunes Hard Law.

Desde este enfoque normativo, las competencias Colombia Compra Eficiente corresponden a meras resoluciones, circulares, directrices, instrucciones, protocolos, normas técnicas e información al público, entre otros (Santos Rodríguez, 2018). Es decir, una serie de herramientas jurídicas que, teóricamente, no constituyen normas obligatorias o actos administrativos con las características que la doctrina tradicional ha concebido; pero sin duda

3 Una de las definiciones que más se acercan y representan el Soft Law es la desarrollada por el tratadista Daniel Sarmiento, de donde toma la información, quien bajo dicho término encierra todos los actos o instrumentos jurídicos sin carácter obligatorio; pero incorporados, de una forma u otra, en el sistema de fuentes (Sarmiento Ramírez - Escudero, 2011). 
producen efectos jurídicos verificables (Martínez Jurado, 2018). Es precisamente el quid de este artículo, cuáles son esos efectos jurídicos, teniendo en cuenta que claramente el Consejo de Estado, Sala de lo Contencioso Administrativo, Subsección A, en la Sentencia del 16 de agosto de 2017, Exp. 56.166 (Sentencia $56166,2017)$ ha establecido que sólo cuando estas herramientas derivan de la ley y sólo en dicho contexto podrán validarse disposiciones reglamentarias que otorguen carácter obligatorio a los instrumentos que esta entidad cree y divulgue.

En este orden de ideas, las facultades otorgadas a las agencias regulatorias considerándolas como emisora de documentos de orientación para el mejor entendimiento y cumplimiento de los procedimientos de adquisición de bienes y servicios del Estado, en especial en lo relacionado con las modalidades de selección reguladas por el Estatuto de la Contratación Pública (Sarmiento Ramírez - Escudero, 2011).

Por ende, la regulación emitida por Colombia Compra Eficiente no cuenta con un carácter de exigibilidad como norma de regulación de la actividad contractual, mucho menos cuando el rango de los instrumentos conlleva por su naturaleza a ser desarrollos interpretativos, sin facultad de ser efectivamente cumplidos o acatados por operadores $\mathrm{y} / 0$ participantes de la actividad contractual (Sarmiento Sánchez, 2015).

Es por esto que las comunicaciones, orientaciones y manuales emitidos por la Agencia Nacional de Contratación tienen, en principio, un efecto vinculante para las entidades sujetas al sistema de adquisición y contratación de obras, bienes y servicios del Estado, es decir, serán de imperativo cumplimiento; mientras no contraríen el ordenamiento vigente y no denoten una extralimitación de las funciones que le fueron otorgadas por el Decreto 4170 de 2011 (Decreto 4170, 2011), permitiendo una correcta práctica y eficiencia de la ley que desarrolla el sistema de compras del Estado (De Vivero Arciniegas, 2013).

A pesar de la postura del Consejo de Estado, que le otorga una potestad reglamentaria a la Agencia Nacional de Contratación Pública, es ostensible el hecho de que los escritos expedidos son de carácter orientador, por ende, su función se caracteriza como una guía en materia de contratación conforme a la naturaleza de las funciones de Colombia Compra Eficiente. Si una entidad estatal realiza un análisis de los lineamientos propuestos por la agencia y determina que no es aplicable o que contrarían la legislación, se les permite apartarse de los mismos y podrá continuar con el proceso de contratación 0 
compras, según los principios y procedimientos establecidos para el caso en concreto que, además de consagrar operaciones más expeditas, posibilitan la materialización de principios como el de selección objetiva, transparencia y pluralidad de oferentes (De Vivero Arciniegas, 2013).

\section{Posición jurisprudencial sobre la influencia de los instrumentos jurídicos emitidos por la Agencia Nacional de Contratación Pública - Colombia Compra Eficiente.}

La jurisprudencia de las Altas Cortes referente al tema sobre el alcance y competencia de la Agencia Nacional es objetivamente escasa. Al respecto se han pronunciado únicamente cuando en el ejercicio de sus funciones la institución ha presentado conflictos con otras entidades estatales y/o leyes.

Tal es el caso de la Sentencia de la Corte Constitucional C 518 de 2016 (Sentencia C 518 de 2016), en la que un ciudadano demanda por inconstitucional algunos apartes del artículo 134 de la Ley 1753 de 2015 (Ley 1753, 2015) "Por la cual se expide el Plan Nacional de Desarrollo 2014-2018 Todos por un nuevo país” por considerar que:

Las normas superiores relacionadas con la supremacía de la Constitución Política, con la naturaleza constitucional de la Comisión Nacional del Servicio Civil, con la autonomía de los Entes Autónomos de creación constitucional, con el principio de unidad de materia y, adicionalmente, con las funciones de administración y vigilancia de los sistemas de carrera a cargo de la Comisión Nacional del Servicio Civil. (Sentencia C 518, 2016)

En la misma, el accionante por medio de tres cargos aduce la inconstitucionalidad de la norma, entre los cuales se encuentra la vulneración a la autonomía propia de la Comisión Nacional del Servicio Civil (en adelante CNSC), ya que en sentencias anteriores la Corte Constitucional definió a la Comisión como un "ente de creación constitucional, autónoma, permanente e independiente del ejecutivo y de las demás ramas del poder público” (Sentencia C 372, 1999) y, por tal motivo, declaró inexequible la injerencia del Departamento Administrativo de la Función Pública en la ejecución de los concursos o procesos de selección que adelanta la CNSC. 
En la mencionada sentencia, la Corte resaltó "la imposibilidad de incluir a una entidad de la rama ejecutiva en el desarrollo de los concursos de méritos adelantados por la CNSC" (Sentencia C 1175, 2005).

Teniendo en cuenta la jurisprudencia aducida, el actor manifestó que las entidades del Estado, especialmente aquellas pertenecientes a la rama ejecutiva del poder público, no pueden reemplazar las atribuciones otorgadas en el Art. 130 de la Carta Política (Constitución Política, 1991) a la CNSC, ya que estas:

Acaban con la posibilidad que tenía de determinar los costos asociados con los concursos o proceso de selección connatural a su objeto, en tanto trasladó dicha función a la Agencia Nacional de Contratación Pública Colombia Compra Eficiente. (Sentencia C 518, 2016).

En su análisis de las disposiciones demandadas la Corte determinan que en lo referente a organismos autónomos como la CNSC:

Los acuerdos marcos desarrollados por el Gobierno Nacional no le resultan obligatorios, de manera que las mismas entidades pueden diseñar sus propios acuerdos marcos a los cuales sujetar los procesos de contratación y, en concreto, de fijación de precios, sin perjuicio de que puedan sujetarse o adherirse a los acuerdos marco realizados por la Agencia Nacional de Contratación Pública - Colombia Compra Eficiente. (Sentencia C 518, 2016)

La Corte continúa manifestando que al no ser obligatorios, no afectan la autonomía de la CNSC y, por tanto, se declaró la exequibilidad de la norma demanda, agregando que:

Tales Acuerdos Marco de Precios no tienen carácter vinculante para la Comisión Nacional del Servicio Civil, quien podrá establecer, diseñar y adoptar sus propios acuerdos, de conformidad con lo previsto en el parágrafo $5^{\circ}$ del artículo 2 de la Ley 1150 de 2007. (Sentencia C 518, 2016).

De manera similar, el Consejo de Estado en su jurisprudencia ha adoptado la noción preceptuada por la Corte Constitucional, como se evidencia en la sentencia 57875 del 13 de octubre de 2017 (sentencia 57875 de 2007), en la que un ciudadano demanda la nulidad la Resolución 1065 de 2016 (Resolución 1065 de 2016) y del acto contentivo del Pliego de Condiciones para seleccionar a los Proveedores de un Acuerdo Marco de Precios para el Servicio de Distribución, 
expedido por la Agencia Nacional de Contratación Pública -Colombia Compra Eficiente para solicitar además la suspensión provisional de dichos actos, al argumentar entre otras situaciones que se violó el artículo 5 de la Ley 1150 de 2007 (Ley 1150, 2007, art. 5), teniendo en cuenta que para la adquisición de bienes y servicios de condiciones técnicas uniformes, el menor precio es el único criterio de selección objetiva válido, además "se violó la competencia de la Comisión de Regulación de Comunicaciones en materia regulatoria de los servicios postales, pues Colombia Compra Eficiente modificó la modalidad de mensajería expresa sin tener facultades para ello” (Sentencia 57875, 2018).

Cabe mencionar que la finalidad del Acuerdo Marco de Precios objeto de la licitación pública de que trata la resolución demandada es lograr la contratación de servicios postales que tienen las características de condiciones técnicas uniformes y común utilización. En consecuencia, tal proceso contractual debe estar sujeto al menor precio, como único criterio de selección objetiva, según lo dispone el artículo 5 de la Ley 1150 de 2007 (Ley 1150, 2007, art. 5). Sin embargo, en el acuerdo se encuentran criterios de evaluación de las ofertas distintos al mencionado.

Adicionalmente, la Ley 1369 de 2009 establece que "La Comisión de Regulación de Comunicaciones -CRC, es la autoridad competente para regular el régimen de tarifas y los niveles de calidad de los Servicios Postales distintos a aquellos pertenecientes al Servicio Postal Universal” (Ley 1369, 2009). No obstante, el pliego objeto de demanda, evidentemente, contraría lo dispuesto por la CRC, al disponer de tiempos de entrega distintos a los estipulados por la Comisión.

Al ser una contradicción notoria, el Consejo de Estado declaró la suspensión provisional de los acápites objeto de discusión, argumentando a su vez que la Agencia Colombia Compra Eficiente se extralimitó en sus funciones, al emitir disposiciones que no son de su competencia y, por ende, al ser violatorios de sus funciones, estos carecen de carácter vinculante.

En ambos casos se observa que las Cortes señalan el carácter no vinculante de las herramientas emitidas por la Agencia Colombia Compra Eficiente. No obstante, según el decreto 4170 de 2011 (Decreto 4170, 2011) y al considerar características específicas de ciertas entidades estatales, sus disposiciones sí poseen este carácter, así lo manifiesta la agencia que: 
Deben aplicar las circulares y los manuales y guías correspondientes de acuerdo con la naturaleza de su régimen y serán de obligatorio cumplimiento cuando hagan referencia a obligaciones que según la normativa del Sistema de Compra Pública deben ser cumplidas por las Entidades Estatales de régimen especial. (Colombia Compra Eficiente, 2017, pág. 1)

Asimismo, manifiesta que los documentos emitidos son obligatorios para todas las entidades estatales cuando están amparados por medio de una circular. En los demás casos, la entidad estatal puede apartarse de las buenas prácticas recogidas por Colombia Compra Eficiente, siempre y cuando no sean aplicables en el caso concreto y se motive tal acción. Particularmente, las circulares externas tienen la característica de ser actos administrativos, es decir, contienen mandatos, orientaciones e instrucciones que van dirigidas al público en general o a las entidades vigiladas, con el fin de dar el conocimiento de la ley y comunicar su oportuna aplicación por parte de ellas (Colombia Compra Eficiente, 2017).

\section{Conclusiones}

Colombia Compra Eficiente hace parte del sector administrativo de planeación, al estar adscrita a su cabeza el Departamento Nacional de Planeación, con las funciones señaladas con anterioridad. Tales funciones corresponden estrictamente a la estructuración de políticas públicas, relativas al tema de la contratación estatal para ser propuestas al Gobierno Nacional.

El marco de acción de la Agencia Nacional de Contratación es limitado, pues a pesar de la clara especificación de sus competencias en su norma de creación, en tanto tiene rango de ley, lo que ha dado pie para que dicha entidad considere que los instrumentos que estructura y difunde en cumplimiento de lo expresamente establecido en su norma de creación tenga carácter vinculante, en la medida en que tengan la naturaleza intrínseca de ser actos administrativos.

Sin embargo, es notorio el hecho de que las entidades estatales consideran que los instrumentos expedidos por la agencia no son de obligatorio cumplimiento sino que estos son conceptos o directrices y, por consiguiente, no poseen carácter vinculante. Es por esto por lo que muchas de estas instituciones omiten las normativas expedidas por la agencia, impiden su aplicación y, como consecuencia, imposibilitan que se cumpla con la finalidad de esta. 


\section{Referencias bibliográficas}

Acosta Ramos, O. A. (2013). La agencia nacional de contratación: Unificación administrativa de la contratación pública colombiana. Trabajo de Grado. Universidad de la Sabana. Obtenido de https://intellectum.unisabana.edu.co/ bitstream/handle/10818/7525/OSCAR\%20ANDRES\%20ACOSTA\%20FINAL. pdf? sequence $=3$ \&isAllowed $=y$

Asamblea Nacional Costituyente. (07 de Julio de 1991). Constitución Política. Colombia: GC 114.

Colombia Compra Eficiente. (29 de Agosto de 2017). Colombia Compra Eficiente. Obtenido de Concepto 4201713000002961: https://sintesis.colombiacompra.gov. co/jurisprudencia/documento/53184

Congreso de la República. (18 de Julio de 2000). Ley 598. Por la cual se crean el Sistema de Información para la Vigilancia de la contratación Estatal, el Catálogo Unico de Bienes y Servicios y el Registro Unico de Precios de Referencia, de los bienes y servicios de uso común en la Administración Pública. DO 44092.

Congreso de la República. (30 de Diciembre de 2009). Ley 1369. Por medio de la cual se establece el régimen de los servicios postales y se dictan otras disposiciones. DO 47578.

Congreso de la República. (09 de Junio de 2015). Ley 1753. Por la cual se expide el Plan Nacional de Desarrollo 2014-2018 "Todos por un nuevo país". DO 49.538.

De Vivero Arciniegas, F. (2013). Las funciones de consulta y expedición de circulares asignadas a la Agencia Nacional de Contratación en el marco de la entrada en vigencia del nuevo Código de Procedimiento Administrativo y de lo Contencioso Administrativo. En J. C. Cassagne, Tratado general de los contratos público (pág. 1175 y s.s.). Buenos Aires: La Ley.

Departamento Nacional de Planeación. (2011). Bases del Plan Nacional de Desarrollo 2010 - 2014: Prosperidad para Todos. Departamento Nacional de Planeación. Obtenido de https://colaboracion.dnp.gov.co/CDT/PND/Bases\%20PND\%2020102014\%20Versi\%C3\%B3n\%205\%2014-04-2011\%20completo.pdf

Departamento Nacional de Planeación. (s.f.). Colombia Compra Eficiente. Obtenido de Colombia Compra Eficiente: https://www.colombiacompra.gov.co/colombiacompra/colombia-compra-eficiente/organigrama

Martínez Jurado, D. (2018). El soft law en derecho administrativo y su control judicial en Colombia. Revista digital de Derecho Administrativo, 1(20), 289 - 343. doi:https:// doi.org/10.18601/21452946.n20.12.

Presidencia de la República. (03 de Noviembre de 2004). Decreto 3620. Por el cual se crea la Comisión Intersectorial de Contratación Pública. DO 45727.

Presidencia de la república. (03 de Noviembre de 2011). Decreto Ley 4170. Por el cual se crea la Agencia Nacional de Contratación Pública- Colombia compra eficiente-, se determinan sus objetivos y estructura. DO 48242.

Santos Rodriguez, J. (2018). Las facultades reglamentarias de la Agencia Nacional de Contratación Pública Colombia Compra Eficiente. Revista digital de Derecho Administrativo, 1(19), 341-347. doi:https://doi.org/10.18601/21452946.n19.14.

Sarmiento Ramirez - Escudero, D. (2011). La Autoridad del Derecho y la Naturaleza del Soft Law. Cuadernos en Derecho Público, 1(28), 221- 266. Obtenido de https:// revistasonline.inap.es/index.php/CDP/article/view/788 
Sarmiento Sanchez, D. E. (2015). La implementación del "Soft Law" o Derecho Blando en el Sistema General de Contratación Estatal. Trabajo de Grado. Bogotá D.C.: Universidad Santo Tomás. Obtenido de https://repository.usta.edu.co/bitstream/ handle/11634/2071/SarmientoDaniel2015.pdf?sequence $=1$

Sentencia 56166, M.P. Jaime Orlando Santofimio Gamboa (Consejo de Estado, Sala de lo Contencioso Administrativo, Subsección A 16 de Agosto de 2017).

Sentencia 57875, M.P.María Adriana Marín. (Consejo de Estado 27 de Septiembre de 2018).

Sentencia C 1175, M.P. Alfredo Beltrán Sierra (Corte Constitucional 17 de Noviembre de 2005).

Sentencia C 372, M.P. José Gregorio Hernandez Galindo (Corte Constitucional 26 de Mayo de 1999).

Sentencia C 518, M.P. Luis Guillermo Guerrero Pérez (Corte Constitucional 21 de Septiembre de 2016). 